\title{
Standardized food challenges are subject to variability in interpretation of clinical symptoms
}

\author{
Francine C van Erp ${ }^{1 *}$, André C Knulst ${ }^{2}$, Yolanda Meijer ${ }^{1}$, Carmelo Gabriele ${ }^{1}$ and Cornelis K van der Ent ${ }^{1}$
}

\begin{abstract}
Background: Food challenge tests are the gold standard in diagnosing food allergy. Guidelines provide scoring systems to classify symptoms during challenge and typically recommend that challenges are considered positive when objective symptoms occur. However, currently no standard criteria for the definition of a positive challenge outcome exists and interpretation of food challenges mainly depends on clinical judgment. This study aims to assess inter- and intra-observer variability in outcomes of routinely performed peanut challenges in children.

Methods: All complete food challenge score sheets of double blind placebo controlled peanut challenges performed in 2008-2010 in an academic hospital were included. Score sheets were reassessed independently by three clinical experts including double reassessment in a subset of score sheets. Inter- and intra-observer variability was evaluated using kappa statistics.
\end{abstract}

Results: We included 191 food challenge score sheets. Inter-observer agreement on overall challenge outcome was moderate $(\mathrm{k}=0.59-0.65)$ and was fair $(\mathrm{k}=0.31-0.46)$ on challenges with symptoms. Intra-observer agreement on overall challenge outcome was good $(k=0.63-0.77)$ but was moderate $(k=0.50-0.60)$ on challenges with symptoms. Subjective symptoms (oral symptoms, abdominal complaints, food aversion) were significantly associated with disagreement between observers.

Conclusions: We demonstrate that, despite strict adherence to guidelines, there is a considerable amount of variability in reassessment of symptoms recorded on food challenges sheets between and within well trained clinicians, especially when subjective symptoms occur.

Keywords: Allergy, Children, Diagnostics, Food challenge, Food allergy, Peanut, Variability

\section{Background}

Food challenge tests are the gold standard in diagnosing food allergy [1]. Several guidelines and symptom score sheets exist to classify symptoms during challenge. A food challenge is usually considered positive when clear objective symptoms occur on verum and not on placebo [1-3]. Whenever possible, symptoms are supported and objectified by measuring clinical parameters such as blood pressure, oxygen saturation and lung function tests. However, no standard criteria for the definition of a positive challenge outcome exist and the interpretation of food challenges mainly depends on clinical judgment. Especially when clear objective symptoms are absent, determination

\footnotetext{
* Correspondence: f.c.vanerp@umcutrecht.nl

'Department of Paediatric Pulmonology and Allergology, Wilhelmina Children's Hospital, University Medical Centre Utrecht, P O Box 85090, 3508, AB, Utrecht, The Netherlands

Full list of author information is available at the end of the article
}

of food challenge outcome can be difficult. Clinicians may then take other factors (course and reproducibility of symptoms over time, patient characteristics, a "gut feeling" or lessons learned from previous cases) into account to determine challenge outcome. These factors come along with clinical judgment in general and are not easily standardized nor implemented in guidelines. Until now no data on the diagnostic accuracy of the interpretation of symptoms during food challenge are available. In this study we describe inter- and intra-observer variability in reassessment of the outcome of previous performed standardized food challenges by measuring the agreement on the outcome of food challenge score sheets.

\section{Methods}

All complete Double Blind Placebo Controlled Food Challenges (DBPCFCs) $(n=191)$ for peanut performed 
in an academic hospital from 2008-2010 were selected for this study. Data were obtained as part of regular patient care and collected retrospectively from electronic patient records in 2012. Food challenge score sheets were used in strictly anonymous form, according to the code of conduct for medical research approved by the hospital's Medical Ethical Committee.

The DBPCFC protocol used in this study was described earlier by Flinterman et al. [4] In short, increasing amounts of defatted peanut flour from 0.01 to $3000 \mathrm{mg}$, were given with time-intervals of 15-30 minutes with randomly dispersed placebo's. Challenges were performed by a nurse practitioner specialized in food allergy and interpreted under supervision of an allergologist. When symptoms occurred the patient was fully examined and in case of doubt or severe symptoms the allergologist was called to interpret these symptoms. All signs and symptoms observed during DBPCFC were recorded in detail on paper food challenge score sheets including timing and administration of doses by a trained nurse and any abnormalities in vital signs (Table 1). Challenges were discontinued and considered positive in case of persistent objective symptoms or if suggestive subjective symptoms (Oral allergy symptoms (OAS)) occurred at 3 subsequent doses or a severe subjective symptom (abdominal pain/nausea with discomfort) lasted for more than 45 minutes. Symptoms

Table 1 Example Food challenge score sheet

\begin{tabular}{|c|c|c|c|}
\hline $\begin{array}{l}\text { Minutes } \\
\text { after start }\end{array}$ & Portion & Time (hr) & Observations/Symptoms \\
\hline \multicolumn{4}{|l|}{ Part 1} \\
\hline $\mathrm{T}=0$ & 1 & 10.30 & $\begin{array}{l}\text { At } 10: 35 \text { patient reports mild } \\
\text { abdominal pain, the pain } \\
\text { disappeared spontaneously } \\
\text { within } 10 \text { minutes. }\end{array}$ \\
\hline $\mathrm{T}=15$ & 2 & 10.45 & - \\
\hline $\mathrm{T}=30$ & 3 & 11.00 & $\begin{array}{l}\text { Patient does not like the food } \\
\text { and eats very slowly. }\end{array}$ \\
\hline $\mathrm{T}=45$ & 4 & 11.15 & - \\
\hline $\mathrm{T}=60$ & 5 & 11.30 & - \\
\hline $\mathrm{T}=75$ & 6 & 11.45 & - \\
\hline $\mathrm{T}=90$ & 7 & 12.00 & $\begin{array}{l}\text { At 12:05 mild sneezing ( } 2 \text { times), } \\
\text { no other symptoms. }\end{array}$ \\
\hline $\mathrm{T}=105$ & 8 & 12.15 & - \\
\hline \multicolumn{4}{|l|}{ Part 2} \\
\hline $\mathrm{T}=120$ & 9 & 12.30 & - \\
\hline $\mathrm{T}=150$ & 10 & 13.00 & $\begin{array}{l}\text { At } 13.15 \text { Severe vomiting (1 time) } \\
\text { No other symptoms. }\end{array}$ \\
\hline $\mathrm{T}=180$ & 11 & NA & - \\
\hline $\mathrm{T}=210$ & 12 & NA & - \\
\hline $\mathrm{T}=240$ & 13 & NA & - \\
\hline
\end{tabular}

Other comments: Patient is a very difficult eater, and did not like the food during challenge. within 15 minutes after a placebo dose were considered as placebo reactions. The three observers were clinical experts in food allergy, regularly interpreted food challenges according to the most recent clinical guidelines [2], had the same criteria for classifying a challenge was positive and worked in close cooperation with each other within an expert centre of food allergy. Observer 1 (a paediatric allergologist) performed food challenges in children for 10 years and supervised included food challenges (2-4 years ago). Observer 2 (a paediatric allergologist in training) performed challenges for more than 5 years. Observer 3 (dermatologist and immunologist) performed food challenges in adults for more than 10 years. Anonymous food challenges score sheets (blinded for patient characteristics, randomization and challenge outcome) were individually administrated to the observers. The observers received $25 \%$ duplicated score sheets randomly dispersed with the other score sheets without their knowledge. They were asked to determine and argue DBPCFC outcome as positive, negative or when information was insufficient or doubtful as inconclusive. Agreement between observers was defined as a concordant classification of all three observers. Disagreement was defined as a discordant classification between two or three observers.

\section{Statistics}

The kappa statistic ( $\mathrm{k}$ ) was used to determine intraobserver and inter-observer variability between different pairs of observers on overall challenge outcomes and on individual symptoms in challenges with symptoms respectively. Interpretation of the Kappa value: $<0.20=$ poor agreement; $0.21-0.40=$ fair agreement; $0.41-0.60=$ moderate agreement; $0.61-0.80=$ good agreement; 0.81 $1.0=$ excellent agreement [5]. For univariable analyses of the association between type of symptoms and the agreement between observers, the chi-square statistic or univariable logistic regression analysis was used. A p-value $<0.05$ was considered statistically significant.

\section{Results}

Initial DBPCFC outcome was positive in 88 (46\%) and negative in 103 (54\%) included challenges. Reactions ranged from Sampson grade 1 to grade 4, only one child showed significant changes in vital signs (tachycardia). Baseline characteristics of children who underwent DBPCFC are shown in Table 2. Agreement of observers with initial challenge outcome ranged from $79 \%-87 \%$. Based on the reassessment of score sheets the observers fully agreed on 132 of 191 (69\%) DBPCFCs, whether the challenge outcome was positive or negative. In 47 (25\%) challenges one observer disagreed with the other two, in 12 (6\%) challenges complete disagreement (negative, positive and inconclusive classification) was present. Inconclusive challenge outcome was recorded by different observers in $58(10 \%)$ reassessments. 
Table 2 Baseline characteristics of children who underwent DBPCFC, $n=191$

\begin{tabular}{ll}
\hline Characteristic & \\
\hline Age, mean (range) in yrs & $7.8(3.4-18.6)$ \\
Male sex, $n(\%)$ & $132(70)$ \\
Peanut slgE, median (IQR) in $\mathrm{KU} / \mathrm{L}$ & $2.60(0.60-18.80)$ \\
Previous reaction to peanut, $n(\%)$ & \\
$\quad$ No ingestion / no reaction & $96(50)$ \\
$\quad$ Non severe & $63(33)$ \\
Severe & $32(17)$ \\
DBPCFC outcome, $n(\%)^{*}$ & \\
Negative & $103(54)$ \\
Grade 1 & $2(1)$ \\
Grade 2 & $51(27)$ \\
Grade 3 & $15(8)$ \\
Grade 4 & $20(11)$ \\
\hline
\end{tabular}

*According to the Sampson classification of anaphylaxis [6].

Reasons reported for inconclusive judgment were insufficient information (50\%), nonspecific symptoms (47\%) or unknown (3\%). Overall 111 (58\%) score sheets could be used to assess inter-observer agreement on individual symptoms. On the remaining 80 (42\%) food challenge score sheets no symptoms were reported.

Results of inter- and intra-observer analysis are shown in Table 3. The inter-observer agreement on overall food challenge outcome was moderate with $\mathrm{K}=0.59-0.65$. Analysis of agreement in challenges with symptoms $(\mathrm{n}=111)$ showed only fair agreement between observers, $\mathrm{k}=0.31$ 0.46 . To assess intra-observer variability 48 (25\%) randomly selected duplicated score sheets including 27 (14\%) score sheets with reported symptoms could be used. The intra-observer agreement on overall challenge outcomes in duplicated challenges was, based on the kappa value, relatively good $(\mathrm{K}=0.63-0.77)$. The agreement within observers in challenges with symptoms $(n=27)$ was however moderate, $\mathrm{k}=0.37-0.60$.

Clear objective symptoms (nasal and severe respiratory symptoms and urticaria) were associated with agreement whereas mild objective symptoms (mild respiratory symptoms, eye symptoms, sneezing and skin symptoms other than urticaria) and subjective signs and symptoms (OAS, abdominal complaints and food aversion) were associated with disagreement between observers (Table 4). The more different objective symptoms were present the more agreement between observers was observed (Table 4). The occurrence of subjective symptoms (e.g. abdominal complaints and OAS) was associated with disagreement within observers whereas disagreement was never present when respiratory symptoms occurred (data not shown). Four children (2\%) experienced symptoms on a placebo portion during challenge, observers disagreed on challenge outcome in two of these children. Exclusion of children with placebo reactions did however not change the results of our study (data not shown).

\section{Discussion}

Our results indicate that when presented with the same clinical information about symptoms during food challenges, clinical experts often (in more than 30\%) disagree on food challenge outcome. While this fair amount of disagreement could be seen as disappointing, results could have been expected. It is known from previous studies in other disciplines that variability in interpretation of clinical symptoms is often present, despite the use of guidelines or scoring systems. Investigators of the Paediatric Rome II criteria for diagnosing functional gastrointestinal disorders in children showed low inter observer agreement among gastroenterologists (45\% agreement, $\mathrm{k}=0.4$ ), even when using a standardized symptom scoring system [7]. A study on the agreement between nurses who triaged patients presenting in the emergency

Table 3 Agreement and variability in classification of DBPCFC outcome

\begin{tabular}{|c|c|c|c|c|c|c|}
\hline & \multicolumn{3}{|c|}{ All DBPCFC ( $n=191)$} & \multicolumn{3}{|c|}{ DBPCFC with symptoms $(n=111)$} \\
\hline & 1 & 2 & 3 & 1 & 2 & 3 \\
\hline \multirow[t]{2}{*}{ Agreement with initial DBPCFC outcome } & $79 \%$ & $82 \%$ & $87 \%$ & $65 \%$ & $69 \%$ & $78 \%$ \\
\hline & $1: 2$ & $1: 3$ & $2: 3$ & $1: 2$ & $1: 3$ & $2: 3$ \\
\hline Inter-observer agreement & $78 \%$ & $76 \%$ & $76 \%$ & $76 \%$ & $61 \%$ & $60 \%$ \\
\hline к $(95 \%$ Cl) & $0.65(0.56-0.74)$ & $0.59(0.50-0.68)$ & $0.59(0.50-0.68)$ & $0.46(0.39-0.53)$ & $0.35(0.22-0.48)$ & $0.31(0.25-0.38)$ \\
\hline \multirow[t]{2}{*}{ Overall agreement } & $69 \%$ & & & $50 \%$ & & \\
\hline & 1 & 2 & 3 & 1 & 2 & 3 \\
\hline Intra-observer agreement ${ }^{*}$ & $77 \%$ & $83 \%$ & $88 \%$ & $67 \%$ & $70 \%$ & $81 \%$ \\
\hline к $(95 \%$ Cl) & $0.63(0.45-0.82)$ & $0.71(0.54-0.89)$ & $0.77(0.62-0.92)$ & $0.50(0.37-0.63)$ & $0.52(0.39-0.65)$ & $0.60(0.45-0.60)$ \\
\hline
\end{tabular}

DBPCFC, Double Blind Placebo Controlled Food Challenge.

K, Kappa.

${ }^{*} \mathrm{n}=48$ (All DBPCFC) and $\mathrm{n}=27$ (DBPCFC with symptoms).

Bold numbers express different observers. For example; $\mathbf{1}=$ observer 1 and 1:2 = observer 1 versus observer 2. 
Table 4 Univariate association of symptoms during challenge with observer agreement, $\mathbf{n}=191$

\begin{tabular}{|c|c|c|c|c|}
\hline Tract & Symptoms & Disagree $(n=59)$ & Agree $(n=132)$ & $p$ \\
\hline \multirow[t]{3}{*}{ Upper airways } & Red/itchy eyes & $10(17)$ & $13(10)$ & 0.168 \\
\hline & Sneezing & $10(17)$ & $10(8)$ & 0.056 \\
\hline & Nasal congestion/rhinorrhoea & - & $10(8)$ & $0.043^{*}$ \\
\hline \multirow[t]{5}{*}{ Lower airways } & Cough & $5(9)$ & $6(5)$ & 0.282 \\
\hline & Hoarseness/difficulty swallowing & - & $3(2)$ & 0.243 \\
\hline & In- and/or expiratory stridor & - & $5(4)$ & 0.130 \\
\hline & Wheezing & - & $4(3)$ & 0.177 \\
\hline & Dyspnoea & $3(5)$ & $2(2)$ & 0.153 \\
\hline \multirow[t]{4}{*}{ Gastro-intestinal } & OAS^ & $28(48)$ & $12(9)$ & $0.000^{* *}$ \\
\hline & Abdominal complaints^ & $21(36)$ & $6(5)$ & $0.000^{* *}$ \\
\hline & Vomiting & $4(7)$ & $5(4)$ & 0.374 \\
\hline & Diarrhoea & - & - & - \\
\hline \multirow[t]{5}{*}{ Skin } & Contact urticaria\# & $9(15)$ & $13(9)$ & 0.283 \\
\hline & Redness & $12(20)$ & $9(7)$ & $0.008^{* *}$ \\
\hline & Pruritis & $4(7)$ & $6(5)$ & 0.525 \\
\hline & Urticaria & - & $10(8)$ & $0.043^{*}$ \\
\hline & Angioedema & - & $2(2)$ & 0.342 \\
\hline Neurological & Change in activity level/loss of consciousness & - & - & - \\
\hline \multirow[t]{2}{*}{ Other subjective signs } & Discomfort^ & $2(3)$ & $10(8)$ & 0.283 \\
\hline & Food aversion^ & $14(24)$ & $10(8)$ & $0.003^{* *}$ \\
\hline \multirow[t]{4}{*}{ Number of different objective symptoms } & No objective symptoms & $18(32)$ & $2(4)$ & Ref \\
\hline & 1 symptom & $22(39)$ & $23(40)$ & $0.005^{* *}$ \\
\hline & 2 symptoms & $13(23)$ & $22(40)$ & $0.001 * *$ \\
\hline & 3 symptoms & $3(5)$ & $8(15)$ & $0.002^{* *}$ \\
\hline
\end{tabular}

OAS, Oral Allergy Symptoms; Ref, Reference category.

$\wedge$ Symptoms referred to as subjective symptoms.

\#Local urticaria after direct contact between the challenge material and skin.

* Statistical significant association with agreement.

**Statistical significant association with disagreement.

Bold numbers are statistically significant $(p<0.05)$.

room revealed only $52 \%$ agreement $(\kappa=0.3)$ [8]. Moreover a low level of agreement $(\kappa=0.3)$ among pediatric asthma specialists in classifying asthma serverity according to the $\mathrm{NIH}$ guidelines was found previously [9].

The origin of disagreement between and within observers observed in this study can be explained in several ways. Our results indicate that not the number but the origin and severity of symptoms is related to the amount of disagreement between observers. This is in contrast to previous suggestions that there is less room for doubt about challenge outcome when two or more organ systems are involved or when symptoms are reproducible or persisting [10]. Due to the amount of variability in course of symptoms during challenge between patients, we were unfortunately not able to demonstrate whether the timing of symptoms was related to the level of agreement between observers.
Subjective symptoms or mild objective symptoms (one episode of vomiting or a transient rash) frequently occur in children, usually as the first sign of an allergic reaction during food challenges. However these symptoms can also indicate fear associated with the clinical setting of the challenge or intolerance for the amount of food or the matrix chosen. As mainly subjective symptoms were present in cases on which observers disagreed one could argue that observers have difficulties in the interpretation of food challenge outcome when clear objective symptoms are absent. Moreover, guidelines only provide information on symptoms likely to be associated with positive challenge outcome and can therefore be interpreted and implemented by each observer differently. Reliability of the assessment of food challenges outcome also depends on the information provided. In our study lack of knowledge of the guidelines is unlikely to influence the 
results as all observers were clinical experts in the field of food allergy and used to perform and interpret food challenges. The same clinical information was administered to all observers excluding the possibility of sampling error. Assessment of challenge outcome was based on paper score sheets eliminating the possibility that the interpretation of observers and results of this study were influenced by other (patient related) factors as level of sensitization, age or previous challenge results.

To our knowledge this is the first study exploring agreement between clinical experts in assessing food challenge outcome. Observers reassessed a large number of challenges in a blinded, standardized and accurate way. Due to the retrospective nature there are some limitations that should be considered when interpreting the results of this study. Placebo reactions can influence challenge outcome in young children [11]. DBPCFCs were performed with randomly interspersed placebo's, but observers had only access to blinded score sheets. Unfortunately we were therefore not able to analyse differences between placebo or verum challenges. Challenges were reassessed after two years, based on recorded symptoms during challenge, no additional (photographic or real life) patient information was available. The food challenges score sheet was not validated and lack of information could have caused differences between observers. Based on our results we can therefore not conclude that observers would classify challenge outcome of actual patients in the same manner as they did based on paper score sheets. However it is possible that the lack of agreement we found is even an underestimation of variability in assessment of 'real life' challenges since conditions in this study were standardized in contrast to real life reactions where observers are influenced by many other (patient related) factors.

\section{Conclusion}

Although our study using symptom score sheets might not fully reflect procedures in a real life setting, our observations indicate that different observers may have different opinions about symptoms during food challenge tests. To further investigate whether this variability is also present during real life challenges future prospective studies using an expert panel or for example a scoring system with weightage points for each (type of) symptom to assess food challenge outcomes are needed. To improve standardization of food challenges and diminish variability in interpretation new preferably objective parameters might also be helpful in the future [12-16]. Until now, clinicians should be aware that although experienced and familiar in working according to international guidelines variability in interpretation of food challenge outcome is present when reassessing score sheets of challenges, especially when objective symptoms are absent.

\section{Abbreviations}

DBPCFC: Double blind placebo controlled food challenge; OAS: Oral allergy symptoms.

\section{Competing interests}

The authors declare that they have no competing interests.

\section{Authors' contributions}

FE substantially contributed to design, concept, acquisition of data, analysis and interpretation of data, drafting the article and final approval of version to be published. AK substantially contributed to interpretation of data, drafting the article and revising critically for important intellectual content and final approval of version to be published. CE substantially contributed to design, interpretation of data and revising critically for important intellectual content and final approval of version to be published. YM and CG substantially contributed to interpretation of data, revising critically for important intellectual content and final approval of version to be published. All authors read and approved the final manuscript.

\section{Author details}

'Department of Paediatric Pulmonology and Allergology, Wilhelmina Children's Hospital, University Medical Centre Utrecht, P O Box 85090, 3508 $A B$, Utrecht, The Netherlands. ${ }^{2}$ Department of (Paediatric) Dermatology and Allergology, University Medical Centre Utrecht, Utrecht, The Netherlands.

Received: 28 July 2014 Accepted: 12 November 2014

Published online: 30 November 2014

\section{References}

1. Bindslev-Jensen C, Ballmer-Weber BK, Bengtsson U, Blanco C, Ebner C, Hourihane J, Knulst AC, Moneret-Vautrin DA, Nekam K, Niggemann B, Osterballe M, Ortolani C, Ring J, Schnopp C, Werfel T: Standardization of food challenges in patients with immediate reactions to foods-position paper from the European Academy of Allergology and Clinical Immunology. Allergy 2004, 59:690-697.

2. Sampson H, Gerthvan Wijk R, Bindslev-Jensen C, Sicherer S, Teuber SS, Burks AW, Dubois AEJ, Beyer K, Eigenmann PA, Spergel JM, Werfel T, Chinchilli VM: Standardizing double-blind, placebo-controlled oral food challenges: American Academy of Allergy, Asthma \& ImmunologyEuropean Academy of Allergy and Clinical Immunology PRACTALL consensus report. J Allergy Clin Immunol 2012, 130:1260-1274.

3. Cochrane SA, Salt LJ, Wantling E, Rogers A, Coutts J, Ballmer-Weber BK, Fritsche P, Fernández-Rivas M, Reig I, Knulst A, Le T-M, Asero R, Beyer K, Golding M, Crevel R, Clare Mills EN, Mackie AR: Development of a standardized low-dose double-blind placebo-controlled challenge vehicle for the EuroPrevall project. Allergy 2012, 67:107-113.

4. Flinterman AE, Pasmans SG, Hoekstra MO, Meijer Y, Van Hoffen E, Knol EF, Hefle SL, Bruijnzeel-Koomen CA, Knulst AC: Determination of no-observedadverse-effect levels and eliciting doses in a representative group of peanut-sensitized children. J Allergy Clin Immunol 2006, 117:448-454.

5. Altman DG: Practical Statistics for Medical Research, Volume 12. London: Chapman \& Hall/CRC; 1991

6. Sampson HA: Anaphylaxis and emergency treatment. Pediatrics 2003, $111(6$ Pt 3):1601-1608.

7. Saps M, Di Lorenzo C: Interobserver and intraobserver reliability of the Rome II criteria in children. Am J Gastroenterol 2005, 100:2079-2082.

8. Nakagawa J, Ouk S, Schwartz B, Schriger DL: Interobserver agreement in emergency department triage. Ann Emerg Med 2003, 41:191-195.

9. Baker KM, Brand DA, Hen J: Classifying asthma: disagreement among specialists. Chest 2003, 124:2156-2163.

10. Niggemann B: When is an oral food challenge positive? Allergy 2010 65:2-6.

11. Ahrens B, Niggemann B, Wahn U, Beyer K: Positive reactions to placebo in children undergoing double-blind, placebo-controlled food challenge. Clin Exp Allergy 2014, 44:572-578.

12. Clark AT, Mangat JS, Tay SS, King Y, Monk CJ, White PA, Ewan PW: Facial thermography is a sensitive and specific method for assessing food challenge outcome. Allergy 2007, 62:744-749.

13. Ruëff F, Friedl T, Arnold A, Kramer M, Przybilla B: Release of mast cell tryptase into saliva: a tool to diagnose food allergy by a mucosal challenge test? Int Arch Allergy 2011, 155:282-288. 
14. Glaumann S, Nopp A, Johansson SGO, Borres MP, Nilsson C: Oral peanut challenge identifies an allergy but the peanut allergen threshold sensitivity is not reproducible. PloS one 2013, 8:e53465.

15. Järvinen KM, Sicherer SH: Diagnostic oral food challenges: procedures and biomarkers. J Immunol Meth 2012, 383:30-38.

16. Caffarelli C, Ricò S, Rinaldi L, Povesi Dascola C, Terzi C, Bernasconi S: Blood pressure monitoring in children undergoing food challenge: association with anaphylaxis. Ann Allergy Asthma Immunol 2012, 108:285-286.

doi:10.1186/s13601-014-0043-6

Cite this article as: van Erp et al:: Standardized food challenges are subject to variability in interpretation of clinical symptoms. Clinical and Translational Allergy 2014 4:43.

\section{Submit your next manuscript to BioMed Central and take full advantage of:}

- Convenient online submission

- Thorough peer review

- No space constraints or color figure charges

- Immediate publication on acceptance

- Inclusion in PubMed, CAS, Scopus and Google Scholar

- Research which is freely available for redistribution 